\title{
An Improved Fuzzy Number Approximation using Shadowed Sets
}

\author{
Mohamed A. H. \\ El_Hawy \\ Phd. student faculty of \\ Computer and \\ Information, Cairo \\ University \\ Giza- Egypt
}

\author{
Hesham A. Hassan \\ Faculty of Computer and \\ Information, Cairo \\ University \\ Giza- Egypt
}

\author{
Hesham A. Hefny \\ Institute of Statistical \\ Studies and Research, \\ Cairo University Giza- \\ Egypt
}

\author{
Khaled T. Wassif \\ Faculty of Computer and \\ Information, Cairo \\ University \\ Giza- Egypt
}

\begin{abstract}
The shadowed sets are proposed by Pedrycz as a granule manner to approximate the fuzzy sets with preserving the uncertainty features. Many methods have been made in this context to maintain various characteristics of uncertainty for fuzzy sets. In this paper, a new method is proposed which it is preserve more than one kind of uncertainty in fuzzy sets. The new technique is based on the use of measures of uncertainty directly to induce ideal values of shadowed set. It's more simply and accurate for describing uncertainty. The features of new method are important for decision applications.
\end{abstract}

\section{General Terms}

Soft Computing, Uncertainty, Granular Computing .

\section{Keywords}

Fuzzy sets, Shadowed sets, Fuzziness measure, Nonspecificity measure.

\section{INTRODUCTION}

Fuzzy sets proposed by Zadeh [1] are very important in modeling and process vague information. Membership functions play vital role to describe vagueness and imprecision in linguistic terms. The shadowed sets are proposed by Pedrycz [2] for representing uncertainty in fuzzy sets and simplify computations complexity. In the literature, two other methods proposed to induce shadowed sets. One method [3] constructed shadowed sets based on fuzziness set induced from fuzzy sets and preserve fuzziness measure of fuzzy sets. Another method[4], induced shadowed sets based on combine two interval approximations for values possibly belong to fuzzy sets and another almost surely belong to fuzzy sets. This approach preserves uncertainty of fuzzy sets in the form of expected interval and width of fuzzy sets. In this paper, we will propose a new method to construct shadowed sets using non-specificity measure and fuzziness measure which it is maintenance two types of uncertainty in fuzzy sets. It is also, very simple in calculations. This paper is organized as follows: in section 2, we present brief review about shadowed sets and different methods proposed to induce it. In section 3, we display uncertainty measures of fuzzy sets and types used in every method for construct shadowed sets. In section 4, we display proposed approach for building shadowed sets. In section 5 , we use fuzzy numbers examples to apply new method to illustrate the proposed algorithm and discussion the new method with previous methods. Finally conclusions have been evolved in section 6 .

\section{SHADOWED SETS $[2,5]$}

Shadowed sets are information granules construct from fuzzy set. The main features of this set are capturing the essence of fuzzy sets, reducing the computational costs and more abilities of interpretation. [6]. Let fuzzy set $\tilde{\mathrm{F}}$, forming shadowed set $\mathrm{S}$ as the equation (1) and figure 1

$$
S: X \rightarrow\{0,1,[0,1]\}
$$

For preserving the uncertainty modeled by fuzzy sets, the process of creating shadowed set start by selecting the threshold $\alpha \in(0,0.5)$ that constructing three regions as illustrated in figure 2 . First region is induced by reduce all membership values less than the threshold $\alpha$ to 0 . Second region forming by elevated membership values more than 1- $\alpha$ to 1 and last region is unknown membership values or shadow regions for membership values around 0.5 as illustrated in figure 2. Selecting $\alpha$ depend on balance of uncertainty with these regions in the form of following equation

$$
v\left(r_{1}\right)+v\left(r_{2}\right)=v\left(r_{3}\right)
$$

Where $v$ is uncertainty of regions $r_{1}, r_{2}, r_{3}$. This balance is achieved by minimization of performance index for the threshold $\alpha$ as the following equation.

$$
V_{\alpha}=\left|v\left(r_{1}\right)+v\left(r_{2}\right)-v\left(r_{3}\right)\right|
$$

Pedrycz calculated optimum $\alpha$ for triangular, Gaussian and parabolic fuzzy sets to be $0.4142,0.395$ and 0.405 respectively.

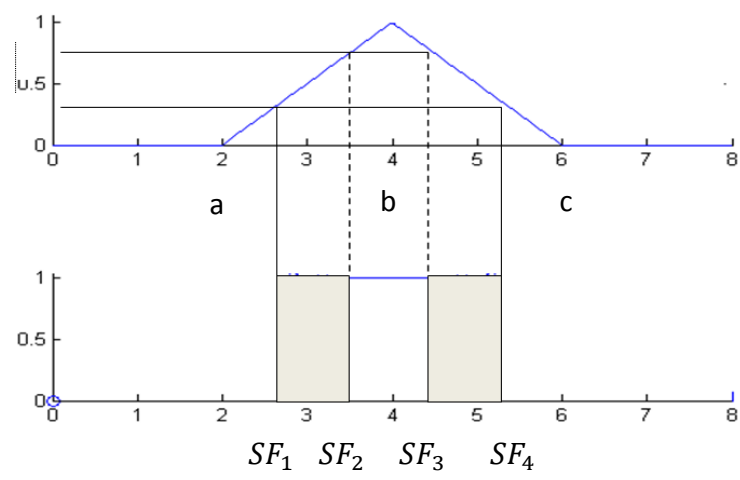

Fig 1: Shadowed sets induced from triangular fuzzy number 


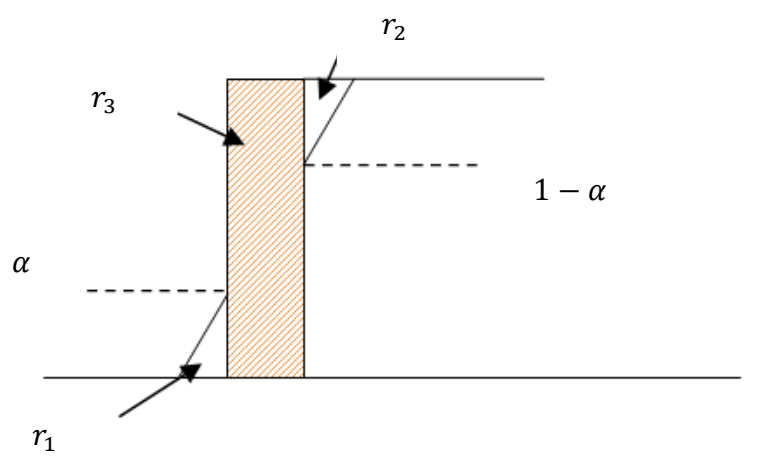

Fig 2 : Regions that construct shadowed set

For example Let $\tilde{F}_{1}=\left(a_{1}, b_{1}, c_{1}\right)$ be triangular fuzzy number then shadowed set $S F^{1}$ induced from it with $\alpha=0.4142$ as illustrated in figure 1 is defined as

$$
\begin{gathered}
S F_{1}^{1}=a_{1}+\alpha\left(b_{1}-a_{1}\right), \\
S F_{2}^{1}=a_{1}+(1-\alpha)\left(b_{1}-a_{1}\right), \\
S F_{3}^{1}=b_{1}+\alpha\left(c_{1}-b_{1}\right), \\
S F_{4}^{1}=b_{1}+(1-\alpha)\left(c_{1}-b_{1}\right)
\end{gathered}
$$

\subsection{Grzegorzewski method [4]}

Grzegorzewski proposed a new algorithm for approximation shadowed sets based on observation of shadowed sets is conjunction of two intervals. First interval, the values possibly belong to fuzzy number as $\left[S F_{1}^{i}, S F_{4}^{i}\right]$ in figure 1 . Second, the elements that almost surely belonging to fuzzy number as $\left[S F_{2}^{i}, S F_{3}^{i}\right]$. Formally, Let $\widetilde{F}$ is a fuzzy number then find two intervals approximations $\bar{S}, \overline{\bar{S}}$ where $\bar{S}=\left[\bar{S}_{L}, \bar{S}_{U}\right]$ and $\overline{\bar{S}}=\left[\overline{\bar{S}}_{L}, \overline{\bar{S}}_{U}\right]$ that composed a shadowed set approximation nearest to fuzzy number $\tilde{F}$ where

$$
\begin{gathered}
\overline{\bar{S}}_{L}=2 \int_{0}^{1} \tilde{F}_{L}(\alpha) d \alpha-2 \int_{0}^{1} \alpha \tilde{F}_{L}(\alpha) d \alpha, \\
\bar{S}_{L}=2 \int_{0}^{1} \alpha \tilde{F}_{L}(\alpha) d \alpha, \\
\bar{S}_{U}=2 \int_{0}^{1} \alpha \tilde{F}_{U}(\alpha) d \alpha, \\
\overline{\bar{S}}_{U}=2 \int_{0}^{1} \tilde{F}_{U}(\alpha) d \alpha-2 \int_{0}^{1} \alpha \tilde{F}_{U}(\alpha) d \alpha
\end{gathered}
$$

and a shadowed set $S(\tilde{F})=\left(s_{1}, s_{2}, s_{3}, s_{4}\right)=\left(\overline{\bar{S}}_{L}, \bar{S}_{L}, \bar{S}_{U}, \overline{\bar{S}}_{U}\right)$. This approach has some features that better than Pedrycz method. First, this approximation is suggested a direct and general formulae for parameters of shadowed sets for any type of fuzzy number. Second, it is preserves the expected interval and width of fuzzy numbers that better for represent uncertainty.

\subsection{Tahayori method [3]}

Tahayori and et al. proposed a new algorithm for the construction of shadowed sets. The main feature of this method is preserving the fuzziness of fuzzy sets. The fuzziness set $f_{A}$ is installed as the following

$$
f_{A}=\left(u_{i}, f u z z\left(u_{i}\right)\right)
$$

Fuzziness set is building based on the following measure of fuzziness.

$$
\operatorname{fuzz}\left(u_{i}\right)=\left[1-\left|2 \mu_{A}\left(u_{i}\right)-1\right|\right]
$$

The second step is deriving the gradual number of cardinality of fuzziness set using assignment function $C_{f_{A}}^{*}(\alpha)$ where.

$$
C_{f_{A}}^{*}(\alpha)=\operatorname{Card}\left(\left(\mathrm{f}_{\mathrm{A}}\right)_{\alpha}\right),
$$

$\left(\mathrm{f}_{\mathrm{A}}\right)_{\alpha}$ is $\alpha$-cut of fuzziness set.

The third step is calculating the threshold $\beta$ such that the membership values of all elements with $\mu_{f_{A}}\left(u_{i}\right)<\beta$ would compensate the membership values of all elements with $\mu_{f_{A}}\left(u_{i}\right) \geq \beta$ to become full members of $\mathrm{f}_{\mathrm{A}}$ as the following.

$$
\sum_{\mu_{f_{A}}\left(u_{i}\right)<\beta} \mu_{f_{A}}\left(u_{i}\right)=\sum_{\mu_{f_{A}}\left(u_{i}\right) \geq \beta}\left(1-\mu_{f_{A}}\left(u_{i}\right)\right)
$$

Or as in figure (3). $d_{1,1}+d_{1,2}=d_{2,1}+d_{2,2}$
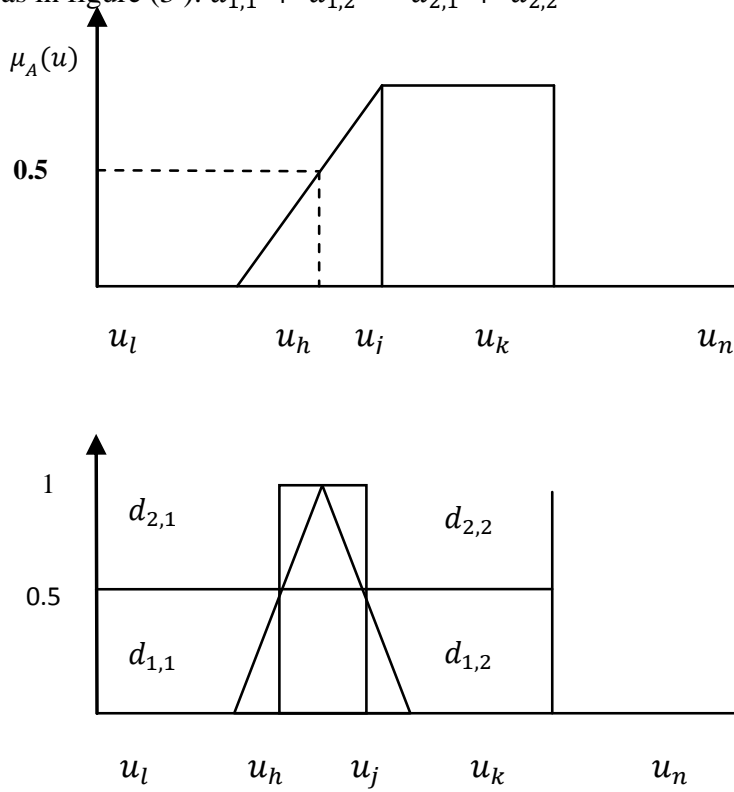

Fig 3 :Fuzziness set

After capture $\beta$ value for fuzziness set of A, all membership values where $\mu_{f_{A}}\left(u_{i}\right) \geq \beta$ are elevated to 1 . Also, all membership values where $\mu_{f_{A}}\left(u_{i}\right)<\beta$ are reduced to 0 . This new area represents cardinality of fuzziness set $f_{A}$. The threshold $\alpha$ calculate where.

$$
\beta=2 \alpha
$$

The previous step can be achieved through gradual fuzziness number. The authors argue that $\exists r \in R$, such that.

Where

$$
\sum_{r \geq C_{f_{A}}^{*}(\delta)}\left(r-C_{f_{A}}^{*}(\delta)\right)=\sum_{r \leq C_{f_{A}}^{*}(\delta)}\left(C_{f_{A}}^{*}(\delta)-r\right)
$$

$$
r=\frac{\sum_{\delta \epsilon L^{+}}\left(C_{f_{A}}^{*}(\delta)\right)}{\left|L^{+}\right|},
$$

$L^{+}$the domain levels of total assignment function. The shadowed set of A can calculate by the threshold $\beta$,

$$
\forall \beta \in C_{f_{A}}^{*^{-1}}\left(\frac{\sum_{\delta \epsilon L^{+}}\left(C_{f_{A}}^{*}(\delta)\right)}{\left|L^{+}\right|}\right)
$$

\section{UNCERTAINTY MEASURES OF FUZZY SETS}

In the literature, three types of uncertainties defined $[1,7]$ First, fuzziness (or vagueness), results from the imprecise boundaries of fuzzy sets. Second, non-specificity (or imprecision) is related to cardinality of fuzzy sets. Third, strife (or discord), that represent conflicts among the various alternatives sets.

These types are quantified by different measures. 


\subsection{Non-specificity measures}

\subsubsection{Hartley function $[1,7]$.}

Hartley proposed in 1928 uncertainty measure for finite crisp sets of possible alternatives using a class of functions

$$
U(C)=m \cdot \log _{n}|C|
$$

where $|\mathrm{C}|$ is the cardinality of a finite nonempty crisp set $\mathrm{C}$, and $(m>0),(n>1)$ are two positive constants. Uncertainty is measured in bits when $\mathrm{m}=1$ and $\mathrm{n}=2$ and the uncertainty function in this case as the following

$$
U(C)=\log _{2}|C|
$$

In the early of 1980s, the generalized of Hartley function from crisp sets to fuzzy sets is proposed as the following.

$$
U(A)=\frac{1}{h(A)} \int_{0}^{h(A)} \log _{2}\left|A^{\alpha}\right| d \alpha
$$

where $\left|A^{\alpha}\right|$ is the cardinality of the $\alpha$-cut of fuzzy set A and $h(A)$ is the height of fuzzy set $\mathrm{A}$. When the fuzzy set $\mathrm{A}$ is normal and finite fuzzy set, the non-specificity measure is

$$
U(A)=\int_{0}^{1} \log _{2}\left|A^{\alpha}\right| d \alpha
$$

\subsubsection{Width of fuzzy set $[4,8]$}

For preserve uncertainty of fuzzy numbers Grzegorzewski proposed a width of fuzzy numbers as a useful parameter characterizing the non-specificity of a fuzzy number and this measure is defined as

$$
\operatorname{width}(A)=\int_{0}^{1}\left(A_{U}(\alpha)-A_{L}(\alpha)\right) d \alpha
$$

where $\mathrm{A}$ is normal fuzzy set, $A_{U}(\alpha)$ and $A_{L}(\alpha)$ are $\alpha$-cut equations of fuzzy number $\mathrm{A}$

\subsection{Fuzziness measures}

The second class of uncertainty in fuzzy sets is fuzziness. This measure assigns nonnegative real number to fuzzy set $\mathrm{A}$ that reflects the degree of the boundary of fuzzy set A not sharp [1]. There are several types of measures of fuzziness such as entropy measures [9], distance measures between fuzzy set and its complement [10] and other measures [5, 11].

\subsubsection{Entropy measure [9]}

De Luca and et al. defined entropy of finite and discrete fuzzy sets A by

$$
H(A)=-\sum_{i=1}^{n}\left(\mu_{i} \ln \mu_{i}+\left(1-\mu_{i}\right) \ln \left(1-\mu_{i}\right)\right)
$$

Where $\mu_{i}$ is membership value of element $i$.

In the case of continues fuzzy sets support in interval $[\mathrm{a}, \mathrm{b}]$. $H(A)=-\int_{a}^{b}(\mu(x) \ln \mu(x)+(1-\mu(x)) \ln (1-\mu(x)) d x$

\subsubsection{Distance measure[10, 12]}

Yager proposed fuzziness measure based on the difference between fuzzy set and its complement as the following. In the case of discrete fuzzy set A.

$$
f(A)=\sum_{x}\left(1-\left|2 \mu_{A}(x)-1\right|\right)
$$

In the case of continues of fuzzy set A support in interval $[a, b]$.

$$
f(A)=\int_{a}^{b}\left(1-\left|2 \mu_{A}(x)-1\right|\right) d x
$$

\subsection{Uncertainty types and methods of inducing shadowed sets}

In this section, we will present the relation between the types of uncertainty and shadowed sets induced from fuzzy sets using various methods, which previously reported.

\subsubsection{Pedrycz method}

The basic concept for this method is localizing and balancing of uncertainty. The threshold performance $\alpha$ is obtained as in equation (3). It's the main role to eliminate the uncertainty in some area and compensate in intermediate membership for building shadow regions. From equation (3), we note that the type of uncertainty in this method linking to cardinality of fuzzy sets. The cardinality of fuzzy set is a basic structure for non-specificity measure.

This method not preserves full quantity of fuzziness of the fuzzy set [8]. So it's not optimal method to induce shadowed sets.

\subsubsection{Tahayori method}

This method depends on the calculating and keeping fuzziness quantity in the form of fuzziness set. It's translated to shadow area in the shadowed set resulting. The Yager measure is used in this method as in equation (13). So, this method for fuzziness portion only.

\subsubsection{Grzegorzewski method}

The author defined the principle of uncertainty invariance. This concept is about the approximation of one model with another one, this meaning convert uncertainty of one type to another and also, preserving its amount $[4,13]$. The width and expected interval for fuzzy set and shadowed set used to confirm this concept where $[4,8,14]$.

$$
\begin{gathered}
\widetilde{E I}(\operatorname{Sh}(A))=E I(A), \\
\widetilde{w}(\operatorname{Sh}(A))=w(A),
\end{gathered}
$$

Expected interval and width of fuzzy set $\mathrm{A}$ is defined as.

$$
\begin{gathered}
E I(A)=\left[\int_{0}^{1} A_{L}(\alpha) d \alpha, A_{R}(\alpha) d \alpha\right], \\
w(A)=\int_{0}^{1}\left(A_{R}(\alpha)-A_{L}(\alpha)\right) d \alpha \text { and }
\end{gathered}
$$

Expected interval and width of shadowed set $\operatorname{Sh}(A)$ is defined as

$$
\begin{aligned}
& \qquad \widetilde{E I}(\operatorname{Sh}(A))=\left[\frac{s_{1}+s_{2}}{2}, \frac{s_{3}+s_{4}}{2}\right], \\
& \widetilde{W}(\operatorname{Sh}(A))=\left[\frac{s_{3}+s_{4}}{2}-\frac{s_{1}+s_{2}}{2}\right], \\
& \text { Where } \operatorname{Sh}(A)=\left(s_{1}, s_{2}, s_{3}, s_{4}\right)
\end{aligned}
$$

The width of fuzzy number is a measure of non-specificity [4,13].

\section{THE ENHANCEMENT OF SHADOWED SETS}

We note from previous methods that they have one type of uncertainty despite of the fuzzy sets have different types of uncertainty [1]. So we need a new method to get shadowed sets that preserve types of uncertainty of fuzzy sets. In the new method, we try to retain two types of uncertainty (nonspecificity and fuzziness). We note that shadowed sets have 
two basic areas (shadow and core). The shadow area has been used previously to represent fuzziness type of uncertainty [3] and non-specificity in other methods $[2,4]$. The core area used to represent set of elements sure belong to shadowed sets $[2,3,4]$. Klir tried to find the crisp interval, which are characteristic of non-specificity measure of fuzzy sets [7] and then depending on this idea, we propose to induce core area of shadowed sets based non-specificity measure and shadow area based on fuzziness measure. The proposed shadowed set constructs in two phases, the first phase configure core area. The second phase relates to induce fuzziness area.

\subsection{Core interval}

We propose that the core interval induces based on nonspecificity measure. This interval must be equal to nonspecificity value of fuzzy number.

Definition 1. The core interval approximation for fuzzy set A can conclude using the threshold $\alpha$-core that induces from the following equation.

$$
A_{R}(\alpha)-A_{L}(\alpha)+1=2^{H_{A}}
$$

Where $H_{A}$ is the non-specificity Hartley value of fuzzy set A and $A_{L}(\alpha), A_{R}(\alpha)$ are left and right $\alpha$-cut of fuzzy set $\mathrm{A}$. the $\alpha$-core building core interval at level 1 as illustrated in figure (4).
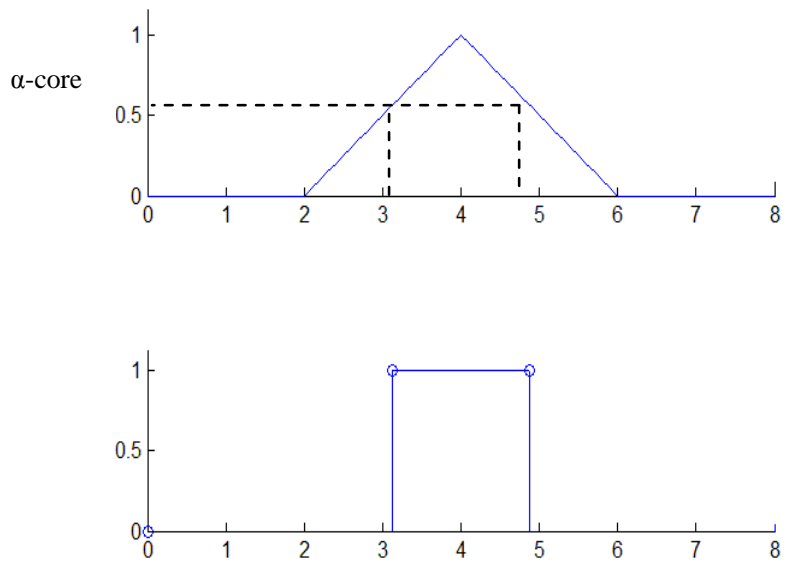

Fig 4 : Core interval for triangular fuzzy set

\subsection{Fuzziness intervals}

Fuzziness sets can be induce using many types of fuzziness measure [8]. In the case of Deluca entropy measure [9]. Let A is a fuzzy set, the fuzziness set $f_{A}$ defines as.

$$
f_{A}=(x, f u z z(x)),
$$

$f u z z(x)=-\mu(x) \ln \mu(x)-(1-\mu(x)) \ln (1-\mu(x))$.

The fuzziness set of convex and normal fuzzy set A consists of left and right fuzziness s sets as in figure 5 .
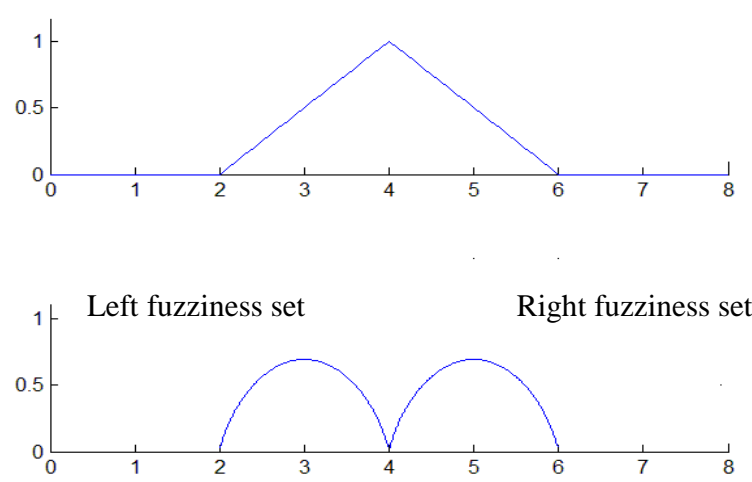

Fig 5 : Fuzziness set for triangular fuzzy set A using entropy measure

We can also use the Yager measure as in figure 6 where the fuzziness sets defined as the following

$$
\begin{aligned}
f_{A} & =(x, f u z z(x)), \\
f u z z & (x)=1-\left|2 \mu_{A}(x)-1\right| .
\end{aligned}
$$
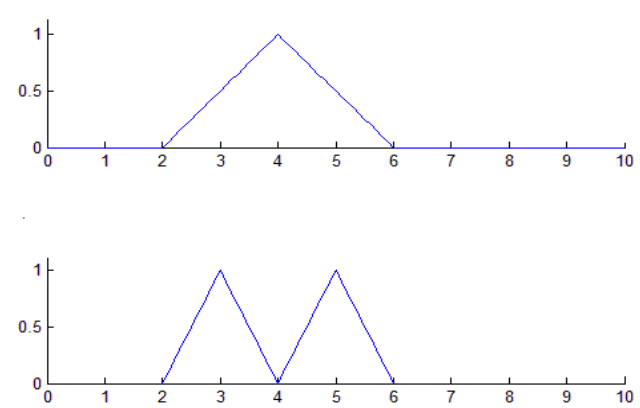

Fig 6 : Fuzziness set for triangular fuzzy set A using Yager measure

The fuzziness set of convex and normal fuzzy set A consists of left and right fuzziness sets. The widths of left and right fuzziness areas calculate as the following.

$$
\begin{aligned}
& w_{L}=\int_{x_{L}} f u z z\left(x_{L}\right), \\
& w_{R}=\int_{x_{R}} f u z z\left(x_{R}\right)
\end{aligned}
$$

By using the widths $w_{L}, w_{R}$, the left and right fuzziness intervals are represented from core points of fuzzy set as in figure (7).

In this stage, we transform left and right fuzziness set to two intervals that start from core points of fuzzy set, as in figure (7). Fuzziness intervals are uncertainty regions. The area of left and right regions of intervals represents cardinality of left and right fuzziness sets. 

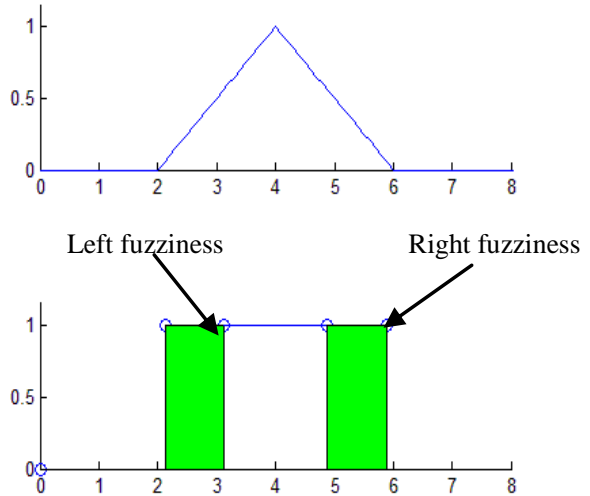

Fig 7: Fuzziness intervals for triangular fuzzy set

\subsection{Shadowed fuzzy number}

When shadowed set induced from fuzzy numbers the shadowed sets called shadowed fuzzy numbers (SFN) [15] as in figure (8). We propose to define with the following parameters.

$$
\operatorname{SFN}(A)=\left(F_{L}, C_{L}, C_{R}, F_{R}\right) ; \text { core }_{A}
$$

Where $\left[F_{L}, C_{L}\right],\left[C_{R}, F_{R}\right]$ are left and right fuzziness intervals and $\left[C_{L}, C_{R}\right]$ is core interval. core $_{A}$ is core value of fuzzy set A. In the case of trapezoidal fuzzy set, the core value is interval.

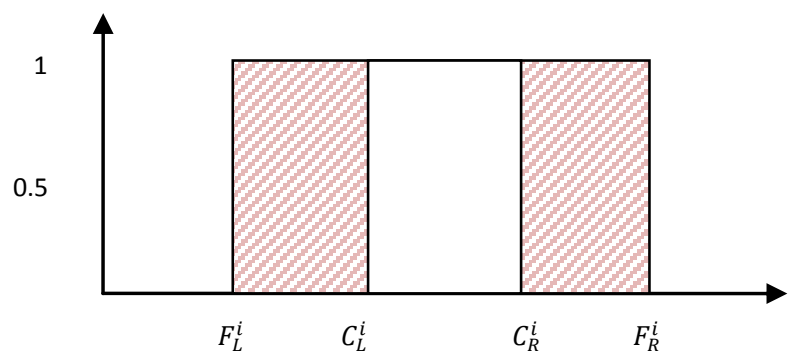

Fig 8 :Shadowed fuzzy number

\subsection{Measures of uncertainty for shadowed fuzzy number}

In this section, we will use the following measures of uncertainty for new shadowed fuzzy numbers. Let $\operatorname{SFN}(A)=$ $\left(F_{L}, C_{L}, C_{R}, F_{R}\right)$; $_{\text {core }}$ is shadowed fuzzy number, we propose the following measures. The Hartley measure of nonspecificity for shadowed fuzzy number as

$$
H(\operatorname{SFN}(A))=\log _{2}\left(C_{R}-C_{L}+1\right)
$$

The measure of fuzziness as.

$$
\operatorname{Fuzz}(\operatorname{SFN}(A))=\left(C_{L}-F_{L}\right)+\left(C_{R}-F_{R}\right)
$$

The width of shadowed fuzzy number as

$$
W(\operatorname{SFN}(A))=\frac{\left(\operatorname{core}_{A}+F_{R}\right)}{2}-\frac{\left(\operatorname{core}_{A}+F_{L}\right)}{2}
$$

We apply these measures for different methods for building shadowed fuzzy numbers.

\section{COMPARISON BETWEEN NEW METHOD AND PREVIOUS METHODS}

In this section, we will present features of new technique and compare it with previous methods through four examples for different types of fuzzy numbers.

Example 1: Let $A$ is a triangular fuzzy number with three parameters $(2,4,6)$. Using our method, we obtain nonspecificity value $H_{A}=1.64$ as equation (20). Then by applying value $H_{A}$ in equation (35), the $\alpha$-core $=0.56$ value result where it is used to induce core interval [3.12, 4.88]. The second step, we use equations (40), (41) to obtain cardinality of left and right fuzziness set. If Yager fuzziness measure used as (28) the left and right cardinality are 1, 1. If entropy fuzziness measure used as (26) the left and right cardinality of fuzziness set are 1,1 . By using cardinality values to calculate the width of left and right fuzziness intervals. The result shadowed fuzzy number is $(2.12,3.12,4.88,5.88)$ as illustrated in figure 7. Using previous methods to solve this example, the shadowed fuzzy number SFN as Pedrycz method is $(2.83,3.17,4.83,5.17) ;$ core $_{A}=4$, using Tahayori method SFN is $(2.5,3.5,4.5,5.5) ;$ core $_{A}=4$ and in the case of Grzegorzewski method the SFN is $(2.67,3.33,4.67,5.33)$; core $_{A}=4$.

To compare different solutions for example 1, table (1) presents uncertainty measures for fuzzy number $\mathrm{A}$ and

\begin{tabular}{|c|c|c|c|c|}
\hline & $\begin{array}{c}\text { Number } \\
\text { parameters }\end{array}$ & $\begin{array}{c}\text { non- } \\
\text { specificity }\end{array}$ & Width & Fuzziness \\
\hline $\begin{array}{c}\text { Fuzzy } \\
\text { number A }\end{array}$ & $(2,4,6)$ & 1.46 & 2 & 2 \\
\hline $\begin{array}{l}\text { SFN new } \\
\text { method }\end{array}$ & $\begin{array}{l}(2.12,3.12, \\
4.88,5.88)\end{array}$ & 1.47 & 1.88 & 2 \\
\hline $\begin{array}{c}\text { SFN } \\
\text { Pedrycz } \\
\text { method }\end{array}$ & $\begin{array}{l}(2.83,3.17, \\
4.83,5.17)\end{array}$ & 1.41 & 2 & 0.69 \\
\hline $\begin{array}{c}\text { SFN } \\
\text { Tahayori } \\
\text { method }\end{array}$ & $\begin{array}{l}(2.5,3.5, \\
4.5,5.5)\end{array}$ & 1 & 2 & 2 \\
\hline $\begin{array}{c}\text { SFN } \\
\text { Grzegorzew } \\
\text { ski method }\end{array}$ & $\begin{array}{l}(2.67,3.33 \\
4.67,5.33)\end{array}$ & 1.22 & 2 & 1.33 \\
\hline
\end{tabular}
different SFN solutions using equations $(23-24)$,(26),(28), (34), $(43-45)$.

Table 1 : uncertainty measures for example 1.

Example 2: Let B is a trapezoidal fuzzy number with four parameters $(2,4,5,7)$. Using new method the shadowed fuzzy number SFN result is $(2.09,3.09,5.91,6.91) ;$ core $_{B}=[4,5]$ and table 2 shows comparison results with previous methods

Table 2 : uncertainty measures for example 2.

\begin{tabular}{|c|c|c|c|c|}
\hline & $\begin{array}{c}\text { Number } \\
\text { parameters }\end{array}$ & $\begin{array}{c}\text { non- } \\
\text { specificity }\end{array}$ & Width & Fuzziness \\
\hline $\begin{array}{c}\text { Fuzzy } \\
\text { number B }\end{array}$ & $(2,4,5,7)$ & 1.94 & 3 & 2 \\
\hline $\begin{array}{c}\text { SFN new } \\
\text { method }\end{array}$ & $\begin{array}{c}(2.09,3.09, \\
5.91,6.91)\end{array}$ & 1.93 & 2.91 & 2 \\
\hline
\end{tabular}




\begin{tabular}{|c|c|c|c|c|}
\hline $\begin{array}{c}\text { SFN } \\
\text { Pedrycz } \\
\text { method }\end{array}$ & $\begin{array}{c}(2.83,3.17, \\
4.83,6.17)\end{array}$ & 1.87 & 3 & 0.69 \\
\hline $\begin{array}{c}\text { SFN } \\
\text { Tahayori } \\
\text { method }\end{array}$ & $\begin{array}{c}(2.53,3.47, \\
5.53,6.47)\end{array}$ & 1.61 & 3 & 1.88 \\
\hline $\begin{array}{c}\text { SFN } \\
\text { Grzegorz } \\
\text { ewski } \\
\text { method }\end{array}$ & $\begin{array}{c}(2.67,3.33, \\
5.67,6.33)\end{array}$ & 1.73 & 3 & 1.32 \\
\hline
\end{tabular}

Example 3: Let $\mathrm{C}$ is a Gaussian fuzzy number with two parameters $(\mathrm{m}=7, \sigma=1)$. We make the same calculation steps as example 1 and result $\mathrm{SFN}=(5.15,6.19,7.81,8.85)$; core $_{C}=7$. Table 3 shows the results of new method compared to previous methods.

Table 3 : uncertainty measures for example 3.

\begin{tabular}{|c|c|c|c|c|}
\hline & $\begin{array}{c}\text { Number } \\
\text { parameters }\end{array}$ & $\begin{array}{c}\text { non- } \\
\text { specificity }\end{array}$ & Width & Fuzziness \\
\hline $\begin{array}{c}\text { Fuzzy } \\
\text { number C }\end{array}$ & $\begin{array}{l}(\mathrm{m}=7 \\
\sigma=1)\end{array}$ & 1.4 & 1.78 & 2.08 \\
\hline $\begin{array}{c}\text { SFN new } \\
\text { method }\end{array}$ & $\begin{array}{l}(5.15,6.19, \\
7.81,8.85)\end{array}$ & 1.4 & 1.85 & 2.08 \\
\hline $\begin{array}{c}\text { SFN } \\
\text { Pedrycz } \\
\text { method }\end{array}$ & $\begin{array}{l}(6.04,6.29 \\
7.71,7.96)\end{array}$ & 1.27 & 2.66 & 0.51 \\
\hline $\begin{array}{c}\text { SFN } \\
\text { Tahayori } \\
\text { method }\end{array}$ & $\begin{array}{c}(5.77,6.51 \\
7.5,8.24)\end{array}$ & 1 & 1.73 & 1.48 \\
\hline $\begin{array}{c}\text { SFN } \\
\text { Grzegorz } \\
\text { ewski } \\
\text { method }\end{array}$ & $\begin{array}{l}(5.85,6.37, \\
7.63,8.15)\end{array}$ & 1.17 & 1.78 & 1.04 \\
\hline
\end{tabular}

Example 4: Let D is a general fuzzy number has the following membership function [16]

$$
\mu_{D}(x)=\left\{\begin{array}{lr}
1-\left(\frac{x-5}{2}\right)^{2} & \text { if } 3 \leq x \leq 7 \\
0 & \text { otherwise. }
\end{array}\right.
$$

with three parameters $(3,5,7)$. Using new method the $\mathrm{SFN}=$ $(2.95,3.73,6.27,7.05) ;$ core $_{D}=5$ and table 4 shows the results of new method compared to previous methods.

Table 4 : uncertainty measures for example 4.

\begin{tabular}{|c|c|c|c|c|}
\hline & $\begin{array}{c}\text { Number } \\
\text { parameters }\end{array}$ & $\begin{array}{c}\text { non- } \\
\text { specificity }\end{array}$ & Width & Fuzziness \\
\hline $\begin{array}{c}\text { Fuzzy } \\
\text { number D }\end{array}$ & $(3,5,7)$ & 1.82 & 2.67 & 1.56 \\
\hline $\begin{array}{c}\text { SFN new } \\
\text { method }\end{array}$ & $\begin{array}{c}(2.95,3.73, \\
6.27,7.05)\end{array}$ & 1.82 & 2.05 & 1.56 \\
\hline
\end{tabular}

\begin{tabular}{|c|c|c|c|c|}
\hline $\begin{array}{c}\text { SFN } \\
\text { Pedrycz } \\
\text { method }\end{array}$ & $\begin{array}{c}(3.39,3.82, \\
6.18,6.61)\end{array}$ & 1.75 & 2.79 & 0.86 \\
\hline $\begin{array}{c}\text { SFN } \\
\text { Tahayori } \\
\text { method }\end{array}$ & $\begin{array}{c}(3.27,4,6, \\
6.73)\end{array}$ & 1.59 & 2.73 & 1.46 \\
\hline $\begin{array}{c}\text { SFN } \\
\text { Grzegorz } \\
\text { ewski } \\
\text { method }\end{array}$ & $\begin{array}{c}(3.4,3.93, \\
6.07,6.6)\end{array}$ & 1.65 & 2.67 & 1.06 \\
\hline
\end{tabular}

\subsection{Discussion of results}

We used in previous examples four different types of fuzzy numbers. We compare the new method and previous methods with regard to retain uncertainty characteristics of fuzzy numbers where three different uncertainty measures are used. We note from the results of previous examples that the new method preserves uncertainty and the best of other methods. In first, second and third examples the new technique preserves three types of uncertainty. In fourth example, two types (non-specificity, fuzziness) are retained. Width and nonspecificity are kept by Pedrycz method for first and second examples and non-specificity for third example. Width and fuzziness are saved by Tahayori method for first example, width for second and third examples and width and fuzziness for fourth example. Width is preserved by Grzegorzewski method for first, second and third examples and two types (non-specificity, width) for fourth example. In view of the optimality, this new method can be the basis to measure the nearest of the result approximation from the best solution.

\section{CONCLUSION}

In this paper, we presented overview of previous methods for building shadowed sets. Also, we presented a brief preview of basic measures of uncertainty and spotting the relation between these measures and shadowed sets result from different approximation methods. We introduced a new method for approximate fuzzy numbers using two measures of uncertainty fuzziness and non-specificity. This new technique tried to keep most of the uncertainty characteristics of fuzzy sets. We present comparative examples between the new approach and previous methods to clarify its accuracy in preserve characteristics of fuzzy sets. From examples results, the new method is superior other than current methods in preserving uncertainty.

\section{REFERENCES}

[1] George. J. Klir, Bo. Yuan, 1995,"Fuzzy Sets and Fuzzy Logic Theory and Applications", Prentice Hall press.

[2] Witold Pedrycz, 1998, "Shadowed Sets: Representing and Processing Fuzzy Sets", IEEE Transactions on systems, man, and cybernetics-part B: Cybernetics, Vol. 28, No. 1, FEBRUARY.

[3] Tahayori, H.; Sadeghian, A.; Pedrycz, W., 2013,"Induction of Shadowed Sets Based on the Gradual Grade of Fuzziness", Fuzzy Systems, IEEE Transactions on , vol.21, no.5, pp.937-949.

[4] P. Grzegorzewski , 2013," Fuzzy number approximation via shadowed sets ", Information Sciences Vol. 225, pp. 35-46. 
[5] Witold Pedrycz, Fernando Gomide, 2007, "Fuzzy systems engineering toward human-centric computing", John Wiley \& Sons press.

[6] Michael Hanss , 2005," Applied Fuzzy Arithmetic: An Introduction with Engineering Applications",Springer Science \& Business Media.

[7] George J. Klir, Mark 1. Wierman, 1999,"Uncertainty Based Information Elements of Generalized Information Theory" Springer-Verlag Berlin Heidelberg GmbH.

[8] S. Chanas, 2001, "On the interval approximation of a fuzzy number", Fuzzy Sets and Systems, Vol.122, pp. 353-356.

[9] A. De Luca, S. Termini, 1972,"A definition of a nonprobabilistic entropy in the setting of fuzzy sets , theory",Information and Control Volume 20, Issue 4, pp. 301-312.

[10] R.R. Yager, 1979, "On the measure of fuzziness and negation, Part I: membership in unit interval", International. J. General Systems, Vol. 5, pp. 221229.

[11] W. Wang and C. Chiu, 1999,"Entropy and information energy for fuzzy sets", Fuzzy Sets and System,
Vol.108,pp. 333-339.

[12] M. Delgado, M.A. Vila, W. Voxman, 1998,"On a canonical representation of a fuzzy number", Fuzzy Sets and Systems, Vol. 93, pp. 125-135.

[13] P. Grzegorzewski, E. Mrowka, 2005,"Trapezoidal approximations of fuzzy numbers", Fuzzy Sets and Systems Vol.153, pp. 115-135.

[14] A. Ban, 2008, "Approximation of fuzzy numbers by trapezoidal fuzzy numbers preserving the expected interval", Fuzzy Sets and Systems, Vol. 159, pp. $1327-1344$.

[15] Olgierd Hryniewicz, , 2006, "An Evaluation of the Reliability of Complex Systems Using Shadowed Sets and Fuzzy Lifetime Data", International Journal of Automation and Computing, Vol. 2 ,pp. 145-150.

[16] P. Grzegorzewski, 2002, "Nearest interval approximation of a fuzzy number", Fuzzy Sets and Systems Vol.130, pp. 321-330. 